\title{
A note on trilinear forms for reducible representations and Beilinson's conjectures
}

Received February 24, 2000 / final version received September 12, 2000

Published online November 8, 2000 - (c) Springer-Verlag \& EMS 2000

Abstract. We extend Prasad's results on the existence of trilinear forms on representations of $G L_{2}$ of a local field, by permitting one or more of the representations to be reducible principal series, with infinite-dimensional irreducible quotient. We apply this in a global setting to compute (unconditionally) the dimensions of the subspaces of motivic cohomology of the product of two modular curves constructed by Beilinson.

\section{Introduction}

Let $F$ be a non-Archimedean local field, and $\pi_{i}(i=1,2,3)$ irreducible admissible representations of $G=G L_{2}(F)$, such that the product of their central characters is trivial. In [8], Prasad shows that there exists, up to a scalar factor, at most one $G$-invariant linear form on $\pi_{1} \otimes \pi_{2} \otimes \pi_{3}$, and determines exactly when such a form exists. These results have been used by Harris and Kudla [6] in the study of the triple product $L$-function attached to three cuspidal automorphic representations of $G L_{2}$ of a global field.

In this note we consider the case when $\pi_{i}$ is permitted to be a reducible principal series representation, whose unique irreducible subspace is infinite-dimensional. It is relatively trivial to extend Prasad's results to cover these cases. The interest in so doing is global. In [1] Beilinson constructs certain subspaces of the motivic cohomology of the product of two modular curves using modular units. His construction can be interpreted as a certain invariant trilinear form on $\pi \otimes \pi^{\prime} \otimes \pi^{\prime \prime}$ taking values in motivic cohomology: here $\pi, \pi^{\prime}$ are weight 2 cuspidal (irreducible) representations of $G L_{2}$ of the finite adeles of $\mathbb{Q}$, and $\pi^{\prime \prime}$ is the space of weight 2 holomorphic Eisenstein series (which is highly reducible). The regulators of these elements of motivic cohomology can be computed as special values of Rankin double product $L$-functions attached to $\pi$ and $\pi^{\prime}$, and Beilinson's calculation of the regulator, together with his general conjectures, predict that these subspaces

M. Harris: Institut de Mathématiques de Jussieu, UMR 7568, Université Paris 7, Denis Diderot, 2 place Jussieu, 75251 Paris Cedex 05, France, e-mail: harris@ math.jussieu.fr

A.J. Scholl: Department of Mathematical Sciences, University of Durham, South Road, Durham DH1 3LE, UK, e-mail: a.j.scholl@durham.ac.uk

Mathematics Subject Classification (1991): 11G40, 11S37 
are one-dimensional. The main aim of the present note is to verify this prediction unconditionally (Theorem 3.1 below).

\section{Local trilinear forms}

Throughout this section, $F$ denotes a non-Archimedean local field, $\mathfrak{o}$ its valuation ring, and $\varpi$ a uniformiser. We let $|-|: F^{*} \rightarrow \mathbb{Q}^{*}$ be the normalised absolute value, so that $|\varpi|^{-1}=\#(\mathfrak{o} / \varpi \mathfrak{o})$. We write $G=G L_{2}(F)$, and denote by $B$ the standard Borel subgroup of upper triangular matrices, by $A$ the diagonal torus, and by $K$ the maximal compact subgroup $G L_{2}(\mathfrak{o})$. As usual $\delta: B \rightarrow \mathbb{Q}^{*}$ denotes the character

$$
\delta\left(\begin{array}{cc}
b_{1} & * \\
0 & b_{2}
\end{array}\right)=\left|\frac{b_{1}}{b_{2}}\right|
$$

(which is the inverse of the modular character of $B$ ). Fix an algebraically closed field $k$ of characteristic zero (in the applications we will take $k=\overline{\mathbb{Q}}$ ), and a square root $\sqrt{p}$ of the residue characteristic of $F$, which determines a square root $\delta^{1 / 2}$ of the character $\delta$. We work in the category of smooth representations of $G$ over $k$. As is customary we do not distinguish between a representation and the space on which it is realised.

We recall standard facts about induced representations of $G$, as can be found in $[4,7]$ or (in much greater generality) in $[2,3,5]$. Let $\mu=\left(\mu_{1}, \mu_{2}\right): A \rightarrow k^{*}$ be a character of $A$, extended to $B$ in the obvious way. Write $\mu^{w}=\left(\mu_{2}, \mu_{1}\right)$. The normalised ${ }^{1}$ induced representation is then

$$
\operatorname{Ind}_{\mathrm{B}}^{\mathrm{G}}(\mu)=\left\{\begin{array}{c}
f: G \rightarrow k \text { locally constant s.t. } \\
f(b g)=\mu(b) \delta(b)^{1 / 2} f(g) \text { for all } b \in B, g \in G
\end{array}\right\} .
$$

This is an admissible representation of $G$ which is indecomposable. It is irreducible if and only $\mu_{1} \mu_{2}^{-1} \neq|-|^{ \pm 1}$, in which case it is also isomorphic to $\operatorname{Ind}_{\mathrm{B}}^{\mathrm{G}} \mu^{w}$. If it is reducible we may assume, twisting by a character of $F^{*}$ if necessary, that $\mu=\delta^{ \pm 1 / 2}=\left(\mu^{-1}\right)^{w}$, and there are then non-split exact sequences of $G$-modules

$$
\begin{aligned}
& 0 \rightarrow k \rightarrow \operatorname{Ind}_{\mathrm{B}}^{\mathrm{G}}\left(\delta^{-1 / 2}\right) \rightarrow \mathrm{Sp} \rightarrow 0 \\
& 0 \rightarrow \mathrm{Sp} \rightarrow \operatorname{Ind}_{\mathrm{B}}^{\mathrm{G}}\left(\delta^{1 / 2}\right) \stackrel{\ell}{\longrightarrow} k \rightarrow 0
\end{aligned}
$$

where Sp, the special or Steinberg representation, is the representation of $G$ acting on the space of locally constant functions on $\mathbb{P}^{1}(F)=B \backslash G$ modulo constant functions. The space of $K$-invariants of each of the representations $\operatorname{Ind}_{\mathrm{B}}^{\mathrm{G}} \delta^{ \pm 1 / 2}$ is one-dimensional: for $\operatorname{Ind}_{\mathrm{B}}^{\mathrm{G}} \delta^{-1 / 2}$ it is the $G$-invariant subspace of constant functions; for $\operatorname{Ind}_{\mathrm{B}}^{\mathrm{G}} \delta^{1 / 2}$ it is the subspace spanned by the function $\phi: b k \mapsto \delta(b)$ (for $b \in B, k \in K)$, and the linear form $\ell$ in (1.2) can be normalised so that $\ell(\phi)=1$. Recall also that $\mathrm{Sp}$ is its own contragredient, and that $\operatorname{dim} \mathrm{Sp}^{K_{0}(\varpi)}=1$, where $K_{0}(\varpi)$ denotes the Iwahori subgroup (elements of $K$ which are congruent $\bmod \varpi$

\footnotetext{
1 It would be preferable to use unnormalised induction, but we refrain from doing so in order to be able to quote from [8] without confusion.
} 
to an element of $B$ ). It follows that the $G$-invariant form $\mathrm{Sp} \otimes \mathrm{Sp} \rightarrow k$ is symmetric, because it must be non-zero on $\mathrm{Sp}^{K_{0}(\varpi)} \otimes \mathrm{Sp}^{K_{0}(\varpi)}$. (The same holds for any irreducible admissible representation of $G$ with trivial central character by the theory of newvectors, an observation of Prasad and Ramakrishnan).

If $\pi$ is an irreducible admissible representation of $G$, its central character will be denoted $\omega_{\pi}$.

Write $G^{\prime}$ for the group of invertible elements of the unique quaternion division algebra over $F$. If $\pi$ is a square-integrable (= discrete series) irreducible admissible representation of $G$, let $\pi^{\prime}$ be the irreducible representation of $G^{\prime}$ associated to $\pi$ by the Jacquet-Langlands correspondence [7, §12].

Prasad proves $[8$, Thms 1.1, 1.2, 1.3]

Theorem 1.1. Let $\pi_{i}(1 \leq i \leq 3)$ be irreducible admissible infinite-dimensional representations of $G$ with $\prod \omega_{\pi_{i}}=1$.

(i) If at least one of $\pi_{i}$ is principal series, then

$$
\operatorname{dim} \operatorname{Hom}_{G}\left(\pi_{1} \otimes \pi_{2} \otimes \pi_{3}, k\right)=1 .
$$

(ii) If all of $\pi_{i}$ are discrete series, then

$$
\operatorname{dim} \operatorname{Hom}_{G}\left(\pi_{1} \otimes \pi_{2} \otimes \pi_{3}, k\right)+\operatorname{dim} \operatorname{Hom}_{G^{\prime}}\left(\pi_{1}^{\prime} \otimes \pi_{2}^{\prime} \otimes \pi_{3}^{\prime}, k\right)=1 .
$$

(iii) If all of $\pi_{i}$ are unramified, then the restriction of a non-zero $G$-invariant form on $\pi_{1} \otimes \pi_{2} \otimes \pi_{3}$ to $\pi_{1}^{K} \otimes \pi_{2}^{K} \otimes \pi_{3}^{K}$ is non-zero.

As the Jacquet-Langlands correspondence takes the special representation Sp of $G$ to the trivial representation of $G^{\prime}$, one has:

Corollary 1.2. If $\pi_{1}, \pi_{2}$ are discrete series then

$$
\operatorname{dim} \operatorname{Hom}_{G}\left(\pi_{1} \otimes \pi_{2} \otimes \mathrm{Sp}, k\right)=1 \Longleftrightarrow \pi_{1} \not \tilde{\pi}_{2} .
$$

For convenience we quote two intermediate results from Prasad's paper which we shall need:

Proposition 1.3. [8, Cors. $5.7 \& 5.8]$ For any admissible representation $\pi$ of $G$ and any character $\chi$ of $B$,

$$
\begin{aligned}
& \operatorname{Ext}_{G}^{1}\left(\operatorname{Ind}_{\mathrm{B}}^{\mathrm{G}} \chi, \pi\right)=0 \Longleftrightarrow \operatorname{Hom}_{G}\left(\operatorname{Ind}_{\mathrm{B}}^{\mathrm{G}} \chi, \pi\right)=0 \\
& \operatorname{Ext}_{G}^{1}\left(\pi, \operatorname{Ind}_{\mathrm{B}}^{\mathrm{G}} \chi\right)=0 \Longleftrightarrow \operatorname{Hom}_{G}\left(\pi, \operatorname{Ind}_{\mathrm{B}}^{\mathrm{G}} \chi\right)=0 .
\end{aligned}
$$

Proposition 1.4. [8, p.17] Let $\mu, \mu^{\prime}$ be characters of $A$. Then there is an exact sequence of $G$-modules:

$$
0 \rightarrow c-\operatorname{Ind}_{A}^{G}\left(\mu \mu^{\prime w}\right) \rightarrow \operatorname{Ind}_{\mathrm{B}}^{\mathrm{G}} \mu \otimes \operatorname{Ind}_{\mathrm{B}}^{\mathrm{G}} \mu^{\prime} \rightarrow \operatorname{Ind}_{\mathrm{B}}^{\mathrm{G}}\left(\mu \mu^{\prime} \delta^{1 / 2}\right) \rightarrow 0
$$

where for a character $v: A \rightarrow k^{*}$,

$$
c-\operatorname{Ind}_{A}^{G} v=\left\{\begin{array}{c}
f: G \rightarrow k \text { compactly supported mod } A \text { and locally constant } \\
\text { s.t. } f(a g)=v(a) f(g) \text { for all } a \in A, g \in G
\end{array}\right\} .
$$


We now consider the case when $\pi_{i}$ are admissible representations which are either irreducible or isomorphic to a twist of $\operatorname{Ind}_{\mathrm{B}}^{\mathrm{G}} \delta^{1 / 2}$.

Proposition 1.5. Suppose that $\pi, \pi^{\prime}$ are infinite-dimensional irreducible admissible representations of $G$, with $\omega_{\pi} \omega_{\pi^{\prime}}=1$. Then

$$
\operatorname{dim} \operatorname{Hom}_{G}\left(\pi \otimes \pi^{\prime} \otimes \operatorname{Ind}_{\mathrm{B}}^{\mathrm{G}} \delta^{1 / 2}, k\right)=1 .
$$

Moreover if $\pi$ and $\pi^{\prime}$ are unramified, then the restriction of a non-zero invariant trilinear form to $\pi^{K} \otimes \pi^{\prime K} \otimes\left(\operatorname{Ind}_{\mathrm{B}}^{\mathrm{G}} \delta^{1 / 2}\right)^{K}$ is non-zero.

Proof. For the most part we simply adapt the proofs in [8] - note that the hard case (three supercuspidals) doesn't arise.

Case 1: $\pi$ is supercuspidal.

The analogous case is treated in [8, middle of p.18]. As $\pi$ is supercuspidal, we have by the theory of the Kirillov model $\left.\pi\right|_{B} \simeq c-\operatorname{Ind}_{Z N}^{B} \psi \omega_{\pi}$, and therefore by two applications of Frobenius reciprocity

$$
\begin{aligned}
\operatorname{Hom}_{G}\left(\pi \otimes \pi^{\prime} \otimes \operatorname{Ind}_{\mathrm{B}}^{\mathrm{G}} \delta^{1 / 2}\right) & =\operatorname{Hom}_{G}\left(\pi \otimes \pi^{\prime}, \operatorname{Ind}_{\mathrm{B}}^{\mathrm{G}} \delta^{-1 / 2}\right) \\
& =\operatorname{Hom}_{B}\left(c-\left.\operatorname{Ind}_{Z N}^{B}\left(\psi \omega_{\pi}\right) \otimes \pi^{\prime}\right|_{B}, k\right) \\
& =\operatorname{Hom}_{Z N}\left(\left.\pi^{\prime}\right|_{Z N}, \psi \psi^{-1} \omega_{\pi^{\prime}}\right)
\end{aligned}
$$

and the last group is simply $\operatorname{Hom}_{N}\left(\left.\pi^{\prime}\right|_{N}, \psi^{-1}\right)$ which is 1-dimensional by the existence and uniqueness of the Kirillov model.

(It is worth noting that by [4, Theorem 1.6], $\pi$ is projective in the category of smooth $G$-modules with central character $\omega_{\pi}$, so $\pi \otimes \operatorname{Ind}_{\mathrm{B}}^{\mathrm{G}} \delta^{1 / 2}=\pi \oplus(\pi \otimes \mathrm{Sp})$ and

$$
\operatorname{Hom}_{G}\left(\pi \otimes \pi^{\prime} \otimes \operatorname{Ind}_{\mathrm{B}}^{\mathrm{G}} \delta^{1 / 2}, k\right)=\operatorname{Hom}_{G}\left(\pi \otimes \pi^{\prime}, k\right) \oplus \operatorname{Hom}_{G}\left(\pi \otimes \pi^{\prime} \otimes \operatorname{Sp}, k\right)
$$

which gives a direct proof of 1.2 when at least one of the representations is supercuspidal.)

Case 2: both $\pi$ and $\pi^{\prime}$ are special.

After twisting we can assume that $\pi=\pi^{\prime}=\operatorname{Sp}$. Then as $\operatorname{Hom}_{G}(\operatorname{Sp} \otimes \operatorname{Sp} \otimes \operatorname{Sp}, k)=0$, we get from (1.2)

$$
\operatorname{Hom}_{G}\left(\mathrm{Sp} \otimes \mathrm{Sp} \otimes \operatorname{Ind}_{\mathrm{B}}^{\mathrm{G}} \delta^{1 / 2}, k\right)=\operatorname{Hom}_{G}(\mathrm{Sp} \otimes \mathrm{Sp}, k) \simeq k .
$$

Case 3: $\pi$ principal series, $\pi^{\prime}$ principal series or special.

Suppose $\pi=\operatorname{Ind}_{\mathrm{B}}^{\mathrm{G}} \mu$ where $\mu_{1} / \mu_{2} \neq|-|^{ \pm 1}$. If $\pi^{\prime} \not \approx \tilde{\pi}$, then by Proposition 1.3

$$
\operatorname{Hom}_{G}\left(\pi^{\prime}, \tilde{\pi}\right)=\operatorname{Ext}_{G}^{1}\left(\pi^{\prime}, \tilde{\pi}\right)=0
$$

and by Theorem $1.1, \operatorname{dim}_{H_{0}}\left(\pi^{\prime} \otimes \operatorname{Sp}, \tilde{\pi}\right)=1$. Now by (1.1) we have a long exact sequence 
(1.3) $\quad 0 \rightarrow \operatorname{Hom}_{G}\left(\pi^{\prime}, \tilde{\pi}\right) \rightarrow \operatorname{Hom}_{G}\left(\pi^{\prime} \otimes \operatorname{Ind}_{\mathrm{B}}^{\mathrm{G}} \delta^{1 / 2}, \tilde{\pi}\right)$

$$
\rightarrow \operatorname{Hom}_{G}\left(\pi^{\prime} \otimes \mathrm{Sp}, \tilde{\pi}\right) \rightarrow \operatorname{Ext}_{G}^{1}\left(\pi^{\prime}, \tilde{\pi}\right) .
$$

and therefore $\operatorname{Hom}_{G}\left(\pi \otimes \pi^{\prime} \otimes \operatorname{Ind}_{\mathrm{B}}^{\mathrm{G}} \delta^{1 / 2}, k\right)=\operatorname{Hom}_{G}\left(\pi^{\prime} \otimes \operatorname{Ind}_{\mathrm{B}}^{\mathrm{G}} \delta^{1 / 2}, \tilde{\pi}\right) \simeq k$.

In the case $\pi^{\prime}=\tilde{\pi}$, the exact sequence (1.3) shows that there is at least one nonzero trilinear form. To show it is the only one, we proceed as in $\$ 5$ of [8]; using Proposition 1.4 for $\pi \otimes \operatorname{Ind}_{\mathrm{B}}^{\mathrm{G}} \delta^{1 / 2}$ and then applying the functor $\operatorname{Hom}_{G}(-, \pi)=\operatorname{Hom}_{G}\left(-, \tilde{\pi}^{\prime}\right)$ we get a long exact sequence:

$$
\begin{aligned}
0 \rightarrow \operatorname{Hom}_{G}\left(\operatorname{Ind}_{\mathrm{B}}^{\mathrm{G}} \mu \delta, \pi\right) \rightarrow \operatorname{Hom}_{G}\left(\pi \otimes \operatorname{Ind}_{\mathrm{B}}^{\mathrm{G}} \delta^{1 / 2}, \pi\right) \\
\rightarrow \operatorname{Hom}_{G}\left(c-\operatorname{Ind}_{A}^{G} \mu \delta^{-1 / 2}, \pi\right) .
\end{aligned}
$$

Since $\pi=\operatorname{Ind}_{B}^{G} \mu$ is irreducible, $\operatorname{Hom}_{G}\left(\operatorname{Ind}_{B}^{G} \mu \delta, \pi\right)$ can only be nonzero if $\operatorname{Ind}_{\mathrm{B}}^{\mathrm{G}} \mu \simeq \operatorname{Ind}_{\mathrm{B}}^{\mathrm{G}} \mu \delta$, which means $\mu \delta=\mu^{w}$, forcing $\mu_{1} / \mu_{2}=|-|^{-1}$ which is not the case. Also

$$
\begin{aligned}
\operatorname{Hom}_{G}\left(c-\operatorname{Ind}_{A}^{G} \mu \delta^{-1 / 2}, \pi\right) & =\operatorname{Hom}_{G}\left(c-\operatorname{Ind}_{A}^{G} \mu \delta^{-1 / 2} \otimes \tilde{\pi}, k\right) \\
& =\operatorname{Hom}_{A}\left(\left.\mu \delta^{-1 / 2} \otimes \tilde{\pi}\right|_{A}, k\right)
\end{aligned}
$$

by Frobenius reciprocity, and this last space is one-dimensional by $[8$, Lemma 5.6(a)]. Therefore $\operatorname{dim}_{G}\left(\pi \otimes \operatorname{Ind}_{B}^{G} \delta^{1 / 2}, \pi\right) \leq 1$, and the dimension is therefore exactly one.

For the final statement about unramified representations, we simply go through word-for-word the proof of [8, Thm. 5.10], taking $V_{3}$ (in the notation of loc. cit.) to be $\pi$. The key point is that in the displayed formula in the middle of page 20, the denominator is non-zero; it vanishes only when one of $V_{1}, V_{2}$ is isomorphic to $\operatorname{Ind}_{\mathrm{B}}^{\mathrm{G}} \delta^{-1 / 2}$ (possibly twisted by a quadratic character).

Proposition 1.6. Suppose that $\pi$ is an infinite-dimensional irreducible admissible representation of $G$, with $\omega_{\pi}=1$. Then

$$
\operatorname{dim} \operatorname{Hom}_{G}\left(\pi \otimes \operatorname{Ind}_{\mathrm{B}}^{\mathrm{G}} \delta^{1 / 2} \otimes \operatorname{Ind}_{\mathrm{B}}^{\mathrm{G}} \delta^{1 / 2}, k\right)=1 .
$$

If $\pi$ is unramified then the restriction of any non-zero invariant trilinear form to $\pi^{K} \otimes\left(\operatorname{Ind}_{\mathrm{B}}^{\mathrm{G}} \delta^{1 / 2}\right)^{K} \otimes\left(\operatorname{Ind}_{\mathrm{B}}^{\mathrm{G}} \delta^{1 / 2}\right)^{K}$ is non-zero.

Proof. We have again the exact sequence (1.3) with $\pi^{\prime}=\operatorname{Ind}_{\mathrm{B}}^{\mathrm{G}} \delta^{1 / 2}$, and since $\pi$ is irreducible and not 1-dimensional, $\operatorname{Hom}_{G}\left(\operatorname{Ind}_{\mathrm{B}}^{\mathrm{G}} \delta^{1 / 2}, \tilde{\pi}\right)=0$. By Proposition 1.3 we also have $\operatorname{Ext}_{G}^{1}\left(\operatorname{Ind}_{\mathrm{B}}^{\mathrm{G}} \delta^{1 / 2}, \tilde{\pi}\right)=0$, and by 1.5 we have $\operatorname{dim}_{\operatorname{Hom}}\left(\operatorname{Ind}_{\mathrm{B}}^{\mathrm{G}} \delta^{1 / 2} \otimes\right.$ $\mathrm{Sp}, \tilde{\pi})=1$, giving the result. The proof of the final part is the same as for Proposition 1.5.

For completeness we also show:

Proposition 1.7. $\operatorname{Hom}_{G}\left(\operatorname{Ind}_{\mathrm{B}}^{\mathrm{G}} \delta^{1 / 2} \otimes \operatorname{Ind}_{\mathrm{B}}^{\mathrm{G}} \delta^{1 / 2} \otimes \operatorname{Ind}_{\mathrm{B}}^{\mathrm{G}} \delta^{1 / 2}, k\right)$ is 1-dimensional. It is generated by the form $\ell \otimes \ell \otimes \ell$, which is nonzero on $\left(\operatorname{Ind}_{\mathrm{B}}^{\mathrm{G}} \delta^{1 / 2}\right)^{K} \otimes\left(\operatorname{Ind}_{\mathrm{B}}^{\mathrm{G}} \delta^{1 / 2}\right)^{K} \otimes$ $\left(\operatorname{Ind}_{\mathrm{B}}^{\mathrm{G}} \delta^{1 / 2}\right)^{K}$. 
Proof. Recall (1.2) that $\ell$ denotes a nonzero invariant linear form on $\operatorname{Ind}_{\mathrm{B}}^{\mathrm{G}} \delta^{1 / 2}$, and that there is a unique $K$-fixed vector $\phi \in \operatorname{Ind}_{\mathrm{B}}^{\mathrm{G}} \delta^{1 / 2}$ with $\ell(\phi)=1$. Fix a non-zero invariant form $(-,-): \operatorname{Sp} \otimes \mathrm{Sp} \rightarrow k$. Let $\beta: \operatorname{Ind}_{\mathrm{B}}^{\mathrm{G}} \delta^{1 / 2} \otimes \operatorname{Ind}_{\mathrm{B}}^{\mathrm{G}} \delta^{1 / 2} \otimes \operatorname{Ind}_{\mathrm{B}}^{\mathrm{G}} \delta^{1 / 2} \rightarrow k$ be a $G$-invariant form. Then $\beta$ vanishes on $\mathrm{Sp} \otimes \mathrm{Sp} \otimes \mathrm{Sp}$ by Corollary 1.2. Therefore there are constants $a, b, c \in k$ such that if $v, v^{\prime} \in \operatorname{Sp}$ and $w \in \operatorname{Ind}_{\mathrm{B}}^{\mathrm{G}} \delta^{1 / 2}$, then

$$
\begin{aligned}
& \beta\left(w \otimes v \otimes v^{\prime}\right)=a \ell(w)\left(v, v^{\prime}\right) \\
& \beta\left(v^{\prime} \otimes w \otimes v\right)=b \ell(w)\left(v, v^{\prime}\right) \\
& \beta\left(v \otimes v^{\prime} \otimes w\right)=c \ell(w)\left(v, v^{\prime}\right)
\end{aligned}
$$

Since $\mathrm{Sp}^{K}=0$ we have

$$
\beta(v \otimes \phi \otimes \phi)=0 \quad \text { for all } v \in \mathrm{Sp} .
$$

Put $u_{g}=g \phi-\phi \in \mathrm{Sp}$. Then for any $v \in \mathrm{Sp}$,

$$
\begin{aligned}
0 & =\beta\left(g^{-1} v \otimes \phi \otimes \phi\right)=\beta(v \otimes g \phi \otimes g \phi) \\
& =\beta\left(v \otimes u_{g} \otimes \phi\right)+\beta\left(v \otimes \phi \otimes u_{g}\right)=c\left(v, u_{g}\right)+b\left(u_{g}, v\right)
\end{aligned}
$$

hence $b=-c$ since $(-,-)$ is symmetric. Likewise $b=-a=c$ hence $a=b=$ $c=0$. The vectors $\left\{u_{g} \mid g \in G\right\}$ span Sp over $k$, since $\phi$ is a generator for $\operatorname{Ind}_{\mathrm{B}}^{\mathrm{G}} \delta^{1 / 2}$. Therefore $\beta$ vanishes on all products $u \otimes v \otimes w$ where at least two factors lie in Sp.

It then follows easily from (1.4) that $\beta$ vanishes on all products where at least one factor lies in Sp, which implies that $\beta$ is a multiple of $\ell \otimes \ell \otimes \ell$.

\section{Global trilinear forms}

In this section, $F$ will denote a global field. The symbols $v, w$ will denote finite places of $F$. Let $\mathbb{A}_{f}$ be the ring of finite adeles of $F$ (the restricted direct product of the completions $F_{v}$ over all finite places $v$ ), and $F_{>0}^{*} \subset F^{*}$ the subgroup of elements which are positive at every real place. For each $v$ write $G_{v}=G L_{2}\left(k_{v}\right)$. We use the same notations for objects associated to $G_{v}$ as in the previous section, with a subscript $v$ added.

Write $G_{f}$ for the group $G L_{2}\left(\mathbb{A}_{f}\right)$ (which is the restricted direct product of the local groups $\left.G_{v}\right), B_{f}$ for the upper triangular subgroup of $G_{f}$ and $\delta_{f}=$ $\prod_{v} \delta_{v}: B_{f} \rightarrow \mathbb{Q}^{*}$.

We first consider the passage from local to global forms.

Proposition 2.1. Let $\pi=\otimes^{\prime} \pi_{v}, \pi^{\prime}=\otimes^{\prime} \pi_{v}^{\prime}$, $\pi^{\prime \prime}=\otimes^{\prime} \pi_{v}^{\prime \prime}$ be factorisable admissible representations of $G_{f}$. Assume that each of $\pi_{v}, \pi_{v}^{\prime}$, $\pi_{v}^{\prime \prime}$ is either irreducible or a twist of $\operatorname{Ind}_{B_{v}}^{G_{v}} \delta_{v}^{1 / 2}$. Then

$$
\operatorname{dim} \operatorname{Hom}_{G_{f}}\left(\pi \otimes \pi^{\prime} \otimes \pi^{\prime \prime}, k\right) \leq 1
$$

with equality if and only if for every $v$

$$
\operatorname{dim} \operatorname{Hom}_{G_{v}}\left(\pi_{v} \otimes \pi_{v}^{\prime} \otimes \pi_{v}^{\prime \prime}, k\right)=1 .
$$


Proof. Recall first the definition of the restricted tensor product $\pi=\otimes^{\prime} \pi_{v}$, which depends on a choice of spherical vector $\phi_{v} \in \pi_{v}^{K_{v}}$ for all $v$ outside some finite set $\Sigma$. It is defined to be the inductive limit of finite tensor products $\pi_{S}=\otimes_{v \in S} \pi_{v}$, where $S$ runs over finite sets of places containing $\Sigma$. If $S \subset T$ then the inclusion mapping $\pi_{S} \hookrightarrow \pi_{T}$ is defined by $x \mapsto x \otimes \bigotimes_{v \in T-S} \phi_{v}$. In particular, if

$$
\pi=\bigotimes_{\left\{\phi_{v} \mid v \notin \Sigma\right\}}^{\prime} \pi_{v}, \quad \pi^{\prime}=\bigotimes_{\left\{\phi_{v}^{\prime} \mid v \notin \Sigma\right\}}^{\prime} \pi_{v}^{\prime}, \quad \pi^{\prime \prime}=\bigotimes_{\left\{\phi_{v}^{\prime \prime} \mid v \notin \Sigma\right\}}^{\prime} \pi_{v}^{\prime \prime},
$$

then their tensor product is

$$
\pi \otimes \pi^{\prime} \otimes \pi^{\prime \prime}=\bigotimes_{\left\{\phi_{v} \otimes \phi_{v}^{\prime} \otimes \phi_{v}^{\prime \prime} \mid v \notin \Sigma\right\}}^{\prime} \pi_{v} \otimes \pi_{v}^{\prime} \otimes \pi_{v}^{\prime \prime} .
$$

(Of course it need not be the case that $\left(\pi_{v} \otimes \pi_{v}^{\prime} \otimes \pi_{v}^{\prime \prime}\right)^{K_{v}}$ is 1-dimensional, or even finite-dimensional). To give a non-zero invariant form on $\pi \otimes \pi^{\prime} \otimes \pi^{\prime \prime}$ is therefore equivalent to giving, for each $v$, a non-zero invariant form on $\pi_{v} \otimes \pi_{v}^{\prime} \otimes \pi_{v}^{\prime \prime}$, which for almost all $v$ takes the value 1 on $\phi_{v} \otimes \phi_{v}^{\prime} \otimes \phi_{v}^{\prime \prime}$. Now use Prasad's results (Theorem 1.1) and Propositions 1.5, 1.6 and 1.7. (We have not excluded the possibility that some of the local components of the original representations are one-dimensional, but in that case the local theory is trivial.)

The representations to which 2.1 applies can be highly reducible. We next restrict to a particular class of such representations which (for $F=\mathbb{Q}$ ) arise from weight 2 Eisenstein series. Let $\chi: \mathbb{A}_{f}^{*} / F_{>0}^{*} \rightarrow k^{*}$ be any character of finite order (in other words, $\chi$ is the restriction to $\mathbb{A}_{f}^{*}$ of an idele class character of finite order). Set

$$
\mathcal{I}(\chi)=\left\{\begin{array}{l}
f: G_{f} \rightarrow k \text { locally constant s.t. } f(b g)=\chi\left(b_{1}\right) \delta_{f}(b) f(g) \\
\text { for all } g \in G_{f} \text { and } b=\left(\begin{array}{cc}
b_{1} & * \\
0 & b_{2}
\end{array}\right) \in B_{f}
\end{array}\right\} .
$$

Then $\mathcal{I}(\chi)$ is an admissible $G_{f}$-module and is isomorphic to the restricted tensor product $\otimes_{v}^{\prime} \mathcal{I}_{v}\left(\chi_{v}\right)$, where

$$
\mathcal{I}_{v}\left(\chi_{v}\right)=\operatorname{Ind}_{B_{v}}^{G_{v}}\left(\chi_{v}|-|_{v}^{1 / 2},|-|_{v}^{-1 / 2}\right)
$$

If $\chi_{v}=1$ then $\mathcal{I}_{v}\left(\chi_{v}\right)=\mathcal{I}_{v}(1)=\operatorname{Ind}_{B_{v}}^{G_{v}} \delta_{v}^{1 / 2}$, and we have the exact sequence (1.2):

$$
0 \rightarrow \mathrm{Sp}_{v} \rightarrow \mathcal{I}_{v}(1) \stackrel{\ell_{v}}{\longrightarrow} k \rightarrow 0 .
$$

We assume that when $\mathcal{I}_{v}(1)$ occurs in a restricted tensor product, the associated $K_{v}$-invariant vector $\phi_{v}$ is taken to be the unique one satisfying $\ell_{v}\left(\phi_{v}\right)=1$.

If $\chi=1$ then we have a local linear form $\ell_{v}$ for every $v$, hence their product $\ell_{f}=\otimes^{\prime} \ell_{v}$ is a $G_{f}$-invariant linear form $\ell_{f}: \mathcal{I}(1) \rightarrow k$; we write $\mathcal{I}(1)^{0}=\operatorname{ker} \ell_{f} \subset$ $\mathcal{I}(1)$. If we set

$$
U_{w}=\operatorname{Sp}_{w} \otimes \bigotimes_{v \neq w}^{\prime} \mathcal{I}_{v}(1)
$$

then $\mathcal{I}(1)^{0}$ is the sum of the subspaces $U_{w}$. 
For arbitrary $\chi$, observe that by Chebotarev $\chi_{v}=1$ for infinitely many $v$, so that the global representation $\mathcal{I}(\chi)$ is an admissible $G_{f}$-module of infinite length.

Proposition 2.2. Let $\pi=\otimes^{\prime} \pi_{v}, \pi^{\prime}=\otimes^{\prime} \pi_{v}^{\prime}$ be irreducible admissible representations of $G_{f}$, all of whose local components are infinite-dimensional.

(i) If $\chi: \mathbb{A}_{f}^{*} / F_{>0}^{*} \rightarrow k^{*}$ is any character of finite order and $\omega_{\pi} \omega_{\pi^{\prime}} \chi=1$ then

$$
\operatorname{dim} \operatorname{Hom}_{G_{f}}\left(\pi \otimes \pi^{\prime} \otimes \mathcal{I}(\chi), k\right)=1 .
$$

(ii) If $\pi^{\prime} \not \tilde{\pi}$ and $\omega_{\pi} \omega_{\pi^{\prime}}=1$ then

$$
\operatorname{dim} \operatorname{Hom}_{G_{f}}\left(\pi \otimes \pi^{\prime} \otimes \mathcal{I}(1)^{0}, k\right)=1 .
$$

(iii) If $\pi^{\prime} \simeq \tilde{\pi}$ then

$$
\operatorname{dim} \operatorname{Hom}_{G_{f}}\left(\pi \otimes \pi^{\prime} \otimes \mathcal{I}(1)^{0}, k\right)=\infty .
$$

Proof. (i) This follows immediately from 2.1, 1.1 and 1.5.

(ii) Pick $w$ with $\pi_{w}^{\prime} \not \tilde{\pi}_{w}$. Observe that on the quotient

$$
\mathcal{I}(1) / U_{w}=\bigotimes_{v \neq w}^{\prime} \mathcal{I}_{v}(1)
$$

the subgroup $G_{w} \subset G_{f}$ acts trivially (hence also on $\mathcal{I}(1)^{0} / U_{w}$ ). Therefore $\operatorname{Hom}_{G_{f}}\left(\pi \otimes \pi^{\prime} \otimes \mathcal{I}(1)^{0} / U_{w}, k\right)=\operatorname{Hom}_{G_{f}}\left(\pi \otimes \pi^{\prime} \otimes \mathcal{I}(1) / U_{w}, k\right)=0$, and thus the homomorphisms of restriction

$$
\begin{aligned}
\operatorname{Hom}_{G_{f}}\left(\pi \otimes \pi^{\prime} \otimes \mathcal{I}(1), k\right) \rightarrow \operatorname{Hom}_{G_{f}}\left(\pi \otimes \pi^{\prime} \otimes \mathcal{I}(1)^{0}, k\right) \\
\rightarrow \operatorname{Hom}_{G_{f}}\left(\pi \otimes \pi^{\prime} \otimes U_{w}, k\right),
\end{aligned}
$$

are injective. But the proof of (i) shows that

$$
\operatorname{dim} \operatorname{Hom}_{G_{f}}\left(\pi \otimes \pi^{\prime} \otimes \mathcal{I}(1), k\right)=1=\operatorname{dim}_{H^{\prime}}\left(\pi \otimes \pi^{\prime} \otimes U_{w}, k\right),
$$

so we are done.

(iii) For each $w \notin S$ there is a $G_{f}$-equivariant surjective homomorphism

$$
\begin{aligned}
\lambda_{w}: \mathcal{I}(1) & \rightarrow \mathcal{I}_{w}(1) \\
\otimes^{\prime} x_{v} & \mapsto x_{w} \prod_{v \neq w} \ell_{v}\left(x_{v}\right)
\end{aligned}
$$

where $G_{f}$ acts on $\mathcal{I}_{w}$ via the projection $G_{f} \rightarrow G_{w}$, and whose kernel is

$$
\operatorname{ker} \lambda_{w}=\sum_{w^{\prime} \neq w} U_{w^{\prime}}
$$

Observe that $\lambda_{w}\left(\mathcal{I}(1)^{0}\right)=\operatorname{Sp}_{w} \subset \mathcal{I}_{w}(1)$, and that for any $x \in \mathcal{I}(1)^{0}, \lambda_{w}(x)=0$ for all but finitely many $w$. Therefore the sum of these homomorphisms is a $G_{f}$ - 
equivariant surjection

$$
\lambda=\left(\lambda_{w}\right): \mathcal{I}(1)^{0} \rightarrow \bigoplus_{w} \mathrm{Sp}_{w}
$$

whose kernel is the subspace $\sum_{w \neq w^{\prime}} U_{w} \cap U_{w^{\prime}}$. Therefore we have a $G_{f}$-equivariant surjection

$$
\pi \otimes \pi^{\prime} \otimes \mathcal{I}(1)^{0} \rightarrow \bigoplus_{w} \pi \otimes \pi^{\prime} \otimes \mathrm{Sp}_{w}
$$

Now for all but finitely many $w$ the local components $\pi_{w}, \pi_{w}^{\prime}$ are unramified, hence principal series, so there will exist a nonzero trilinear form on $\pi_{w} \otimes \pi_{w}^{\prime} \otimes \mathrm{Sp}_{w}$. For all $v \neq w$ we have a pairing $\pi_{v} \otimes \pi_{v}^{\prime} \rightarrow k$ by hypothesis. Therefore the right-hand side of (2.2) has an infinite-dimensional quotient on which $G_{f}$ acts trivially.

We also have an analogous result when two of the representations are of the form $\mathcal{I}(\chi)$ or $\mathcal{I}(1)^{0}$ :

Proposition 2.3. Let $\pi=\otimes^{\prime} \pi_{v}$ be an irreducible admissible representations of $G_{f}$ whose local components are all infinite-dimensional. Suppose that $\pi^{\prime}$ and $\pi^{\prime \prime}$ are representations of the form $\mathcal{I}(\chi)$ or $\mathcal{I}(1)^{0}$, and that $\omega_{\pi} \omega_{\pi^{\prime}} \omega_{\pi^{\prime \prime}}=1$. Then

$$
\operatorname{dim} \operatorname{Hom}_{G_{f}}\left(\pi \otimes \pi^{\prime} \otimes \pi^{\prime \prime}, k\right)=1 .
$$

Proof. If both of $\pi^{\prime}, \pi^{\prime \prime}$ are of the form $\mathcal{I}(\chi)$, then this follows from 2.1.

If $\pi^{\prime}=\mathcal{I}(\chi)$ and $\pi^{\prime \prime}=\mathcal{I}(1)^{0}$, then we can choose $w$ such that $\operatorname{Hom}_{G_{w}}\left(\pi_{w} \otimes\right.$ $\left.\mathcal{I}_{w}\left(\chi_{w}\right), k\right)=0$ (it is enough to take $w$ such that $\chi_{w}=1$ and $\pi_{w}$ is unramified). Then the same argument as in 2.2(ii) applies, using 1.6 in place of 1.5.

Finally suppose that $\pi^{\prime}=\pi^{\prime \prime}=\mathcal{I}(1)^{0}$. Then consider the inclusions

$$
U_{w} \otimes \mathcal{I}(1)^{0} \subset \mathcal{I}(1)^{0} \otimes \mathcal{I}(1)^{0} \subset \mathcal{I}(1) \otimes \mathcal{I}(1)^{0}
$$

whose successive quotients are $\left(\mathcal{I}(1)^{0} / U_{w}\right) \otimes \mathcal{I}(1)^{0}$ and $\mathcal{I}(1)^{0}$. We have $\operatorname{Hom}_{G_{f}}\left(\pi \otimes \mathcal{I}(1)^{0}, k\right)=0$. In fact, as $\mathcal{I}(1)^{0}=\sum U_{w}$ it is enough to show that $\operatorname{Hom}_{G_{f}}\left(\pi \otimes U_{w}, k\right)=0$ for every $w$, which is clear locally. We claim that for $w$ such that $\pi_{w}$ is unramified, $\operatorname{Hom}_{G_{f}}\left(\pi \otimes\left(\mathcal{I}(1)^{0} / U_{w}\right) \otimes \mathcal{I}(1)^{0}, k\right)=0$. Again it is enough to show that for every $w^{\prime}, \operatorname{Hom}_{G_{f}}\left(\pi \otimes\left(\mathcal{I}(1)^{0} / U_{w}\right) \otimes U_{w^{\prime}}, k\right)=0$, and this is true locally at $w$, since $\mathcal{I}(1)^{0} / U_{w}$ is trivial at $w$.

For such $w$ the restriction homomorphisms

$$
\begin{aligned}
\operatorname{Hom}_{G_{f}}\left(\pi \otimes \mathcal{I}(1) \otimes \mathcal{I}(1)^{0}, k\right) \rightarrow \operatorname{Hom}_{G_{f}}\left(\pi \otimes \mathcal{I}(1)^{0} \otimes \mathcal{I}(1)^{0}, k\right) \\
\rightarrow \operatorname{Hom}_{G_{f}}\left(\pi \otimes U_{w} \otimes \mathcal{I}(1)^{0}, k\right)
\end{aligned}
$$

are then injective, and Proposition 2.1 and the appropriate local results show that the two outer groups have dimension one. 


\section{Beilinson's subspaces}

We briefly review here Beilinson's results [1] concerning the $L$-function of a product of two modular curves at $s=1$. We use the notation and formulation of [10, §2] where details can be found. For a positive integer $n, M_{n}$ denotes the modular curve over $\mathbb{Q}$ parameterising elliptic curves with full level $n$ structure, and $\bar{M}_{n}$ denotes its smooth compactification. Write $M=\lim _{\longleftarrow} M_{n}, \bar{M}=\lim \bar{M}_{n}$ for the modular curves at infinite level. These are schemes over the maximal abelian extension $\mathbb{Q}^{\text {ab }}$ of $\mathbb{Q}$.

In the notation of the previous section we take $F=\mathbb{Q}$. Then $G_{f}$ acts on $M$ and $\bar{M}$. (We assume that our level structures are defined in such a way that this is a right action). If

$$
K_{n}=\operatorname{ker}\left(G L_{2}(\hat{\mathbb{Z}}) \rightarrow G L_{2}(\mathbb{Z} / n \mathbb{Z})\right)
$$

is the standard level $n$ open compact subgroup of $G_{f}$ then $M_{n}$ is the quotient $M / K_{n}$ and $\bar{M}_{n}=\bar{M} / K_{n}$.

Next recall the decomposition of the motive of a modular curve under the Hecke algebra. We work in the category $\mathcal{M}_{\mathbb{Q}} \otimes \overline{\mathbb{Q}}$ of Chow motives over $\mathbb{Q}$ with coefficients in $\overline{\mathbb{Q}}$. One has a Chow-Künneth decomposition

$$
h\left(\bar{M}_{n}\right)=h^{0}\left(\bar{M}_{n}\right) \oplus h^{1}\left(\bar{M}_{n}\right) \oplus h^{2}\left(\bar{M}_{n}\right) .
$$

The space $\Omega^{1}(\bar{M}) \otimes \overline{\mathbb{Q}}$ of holomorphic weight 2 cusp forms with coefficients in $\overline{\mathbb{Q}}$ decomposes as a direct sum of irreducible admissible representations $\pi$ of $G_{f}$ with multiplicity one. To each such $\pi$ there is associated a rank 2 motive $V_{\pi}$ in $\mathcal{M}_{\mathbb{Q}} \otimes \overline{\mathbb{Q}}$, which is a direct factor of $h^{1}\left(\bar{M}_{n}\right)$ if $\pi^{K_{n}} \neq 0$. The motives $V_{\pi}$ are simple of rank 2 , and $V_{\pi}, V_{\pi^{\prime}}$ are isomorphic if and only if $\pi \simeq \pi^{\prime}$. One then has

$$
h^{1}(\bar{M})=\lim _{\longrightarrow} h^{1}\left(\bar{M}_{n}\right)=\bigoplus_{\pi} V_{\pi} \otimes[\pi] .
$$

Here $V_{\pi} \otimes[\pi]$ means simply the direct sum of an infinite number of copies of $V_{\pi}$, indexed by a basis for $\pi$. It is an ind-object of $\mathcal{M}_{\mathbb{Q}} \otimes \overline{\mathbb{Q}}$ which carries an action of $G_{f}$.

In [1] Beilinson constructs a certain subspace of the motivic cohomology $H_{\mathcal{M}}^{3}\left(\bar{M}^{2}, \mathbb{Q}(2)\right)$ using modular units supported on Hecke correspondences. One has a decomposition

$$
h\left(\bar{M}^{2}\right) \supset h^{1}(\bar{M})^{\otimes 2}=\bigoplus_{\pi, \pi^{\prime}} V_{\pi} \otimes_{\overline{\mathbb{Q}}} V_{\pi^{\prime}} \otimes\left[\pi \times \pi^{\prime}\right]
$$

where $\left[\pi \times \pi^{\prime}\right]$ is the space of the exterior tensor product of $\pi$ and $\pi^{\prime}$. Applying this one can rewrite Beilinson's construction as giving, for each pair $\left(\pi, \pi^{\prime}\right)$, a homomorphism [10, §2.3.3]

$$
\mathrm{Б}\left(\pi \times \pi^{\prime}\right):\left(\mathcal{O}^{*}(M) \otimes_{\mathbb{Z}} \tilde{\pi} \otimes_{\overline{\mathbb{Q}}} \tilde{\pi}^{\prime}\right)_{G_{f}} \rightarrow H_{\mathcal{M}}^{3}\left(V_{\pi} \otimes V_{\pi^{\prime}}, \mathbb{Q}(2)\right)
$$


whose source is the maximal quotient of $\mathcal{O}^{*}(M) \otimes_{\mathbb{Z}} \tilde{\pi} \otimes_{\overline{\mathbb{Q}}} \tilde{\pi}^{\prime}$ on which $G_{f}$ acts trivially.

The $G_{f}$-module $\mathcal{O}^{*}(M) \otimes_{\mathbb{Z}} \overline{\mathbb{Q}}$ can be described almost completely [9]. There is an exact sequence

$$
0 \rightarrow \mathbb{Q}^{\mathrm{a} b *} \otimes_{\mathbb{Z}} \overline{\mathbb{Q}} \rightarrow \mathcal{O}^{*}(M) \otimes_{\mathbb{Z}} \overline{\mathbb{Q}} \rightarrow \mathcal{I}(1)^{0} \oplus \bigoplus_{\chi} \mathcal{I}(\chi) \rightarrow 0
$$

where the direct sum is over all even non-trivial characters $\chi: \mathbb{A}_{f}^{*} / \mathbb{Q}^{*} \rightarrow \overline{\mathbb{Q}}^{*}$ of finite order. The action of $G_{f}$ on the trivial modular units $\mathbb{Q}^{\text {ab* }} \otimes_{\mathbb{Z}} \overline{\mathbb{Q}}$ is the composite of the determinant and the reciprocity law of class field theory.

We now assume that $\pi^{\prime}$ is not isomorphic to a twist of $\pi$; this implies in particular [10, Lemma 2.5.2] that $\mathrm{B}\left(\pi \times \pi^{\prime}\right)$ is trivial on $\mathbb{Q}^{\mathrm{ab} *}$ and [10, Theorem 2.3.4] that its image lies in the integral part of the motivic cohomology, hence factors as

$$
\mathrm{Б}\left(\pi \times \pi^{\prime}\right):\left(\mathcal{I}(\chi)^{0} \otimes \tilde{\pi} \otimes \tilde{\pi}^{\prime}\right)_{G_{f}} \rightarrow H_{\mathcal{M} / \mathbb{Z}}^{3}\left(V_{\pi} \otimes V_{\pi^{\prime}}, \mathbb{Q}(2)\right) .
$$

Here $\chi=\omega_{\pi} \omega_{\pi^{\prime}}$, and if $\chi \neq 1, \mathcal{I}(\chi)^{0} \stackrel{\text { def }}{=} \mathcal{I}(\chi)$. As we shall recall in a moment, one of Beilinson's main results [1, Thm. 6.1.1] shows that $\mathrm{E}\left(\pi \times \pi^{\prime}\right)$ is non-zero. We can then apply Proposition 2.2 to the source of the homomorphism to give:

Theorem 3.1. Assume that $\pi^{\prime}$ is not isomorphic to a twist of $\pi$. Then the image of $\mathrm{Б}\left(\pi \times \pi^{\prime}\right)$ has dimension one.

There is a regulator homomorphism from motivic cohomology to real Deligne cohomology:

$$
r_{\mathcal{H}}: H_{\mathcal{M} / \mathbb{Z}}^{3}\left(V_{\pi} \otimes V_{\pi^{\prime}}, \mathbb{Q}(2)\right) \rightarrow H_{\mathcal{H}}^{3}\left(V_{\pi} \otimes V_{\pi^{\prime}}, \mathbb{R}(2)\right)
$$

whose target is in this case a free $\mathbb{R} \otimes \overline{\mathbb{Q}}$-module of rank one. In $[1, \S 6]$ Beilinson explains how to compute the composite $r_{\mathcal{H}} \circ \mathrm{D}\left(\pi \times \pi^{\prime}\right)$ as a Rankin-Selberg integral; its image is a 1-dimensional $\overline{\mathbb{Q}}$-subspace in $H_{\mathcal{H}}^{3}\left(V_{\pi} \otimes V_{\pi^{\prime}}, \mathbb{R}(2)\right)$, which can be described in terms of the special value $L\left(V_{\pi} \otimes V_{\pi^{\prime}}, 2\right)$. In particular $\mathrm{B}\left(\pi \times \pi^{\prime}\right) \neq 0$, and $\operatorname{dim}_{\overline{\mathbb{Q}}} H_{\mathcal{M} / \mathbb{Z}}^{3}\left(V_{\pi} \otimes V_{\pi^{\prime}}, \mathbb{Q}(2)\right) \geq 1$. Beilinson's general conjectures predict that the dimension is one, but at present even finite-dimensionality is unknown.

It would be nice if the same argument worked for Beilinson's construction of elements of $H_{\mathcal{M}}^{2}\left(V_{\pi}, \mathbb{Q}(2)\right)$. However in this case the generating homomorphism is a $G_{f}$-invariant linear map

$$
\mathrm{Б}(\pi): \mathcal{O}^{*}(M) \otimes \mathcal{O}^{*}(M) \otimes \tilde{\pi} \rightarrow H_{\mathcal{M} / \mathbb{Z}}^{2}\left(V_{\pi}, \mathbb{Q}(2)\right)
$$

When constant units are factored out, its source becomes a direct sum of tensor products

$$
\bigoplus_{\chi \text { even }} \mathcal{I}(\chi)^{(0)} \otimes \mathcal{I}\left(\chi^{-1} \omega_{\pi}\right)^{(0)} \otimes \tilde{\pi}
$$

(where $\mathcal{I}(\chi)^{(0)}$ denotes $\mathcal{I}(1)^{0}$ for $\chi$ trivial, and $\mathcal{I}(\chi)$ otherwise). The space of $G_{f}$-coinvariants of each summand is one-dimensional by Proposition 2.3, but this alone does not suffice to bound the image of $\mathrm{B}(\pi)$. 
Acknowledgements. The authors gratefully acknowledge the support of the European Commision through the TMR Network Arithmetic Algebraic Geometry, which enabled this collaboration to take place. The second author also wishes to thank the EPSRC for support during his stay at the Isaac Newton Institute in 1998, when some of the work was done.

\section{References}

1. Beilinson, A.A.: Higher regulators and values of $L$-functions. J. Sov. Math. 30, 2036$2070(1985)$

2. Bernshtein, I.N., Zelevinskii, A.V.: Representations of the group $G L(n, F)$ where $F$ is a non-Archimedean local field. Uspekhi Mat. Nauk 31:3, 5-70 (1976). English translation: Russian Math. Surv. 31:3, 1-68 (1976)

3. Cartier, P.: Representations of $p$-adic groups: a survey. Proc. Symp. Pure Math. AMS 32, 111-155 (1979)

4. Casselman, W.: An assortment of results on representations of $G L_{2}(k)$. In: Modular functions of one variable II. Lect. Notes Math., Vol. 349, pp. 1-54. Berlin, Heidelberg, New York: Springer 1973

5. Casselman, W.: Introduction to the theory of admissible representations of $p$-adic reductive groups. Unpublished notes, posted at http://www. math ubc.ca/people/faculty/cass/

6. Harris, M., Kudla, S.: The central critical value of a triple product $L$-function. Ann. Math. 133, 605-672 (1991)

7. Jacquet, H., Langlands, R.P.: Automorphic forms on $G L(2)$. Lect. Notes Math., Vol. 114. Berlin, Heidelberg, New York: Springer 1970

8. Prasad, D.: Trilinear forms for representations of $G L_{2}$ and local $\epsilon$-factors. Compositio Math. 75, 1-46 (1990)

9. Scholl, A.J.: On modular units. Math. Annalen 285, 503-510 (1989)

10. Scholl, A.J.: Integral elements of $K$-theory and products of modular curves. In: The Arithmetic and Geometry of Algebraic Cycles (ed. B. Brent Gordon et al.). Proceedings of the 1998 NATO Advanced Study Institute. NATO Science Series 548. Dordrecht: Kluwer Academic Press 2000 